\title{
DESIGN AND IMPLEMENTATION OF A RASPBERRY- PI BASED HOME SECURITY AND FIRE SAFETY SYSTEM
}

\author{
Sajid M. Sheikh ${ }^{1}$, Modise K. Neiso ${ }^{2}$ and Fatma Ellouze ${ }^{3}$ \\ ${ }^{1,2}$ Department of Electrical Engineering, Faculty of Engineering and Technology, \\ University of Botswana, Gaborone, Botswana \\ ${ }^{3}$ MIRACL Laboratory, Univeristy of Sfax, Airport Road, BP 1088, 3018 Sfax, Tunisia
}

\begin{abstract}
Fire alarms and building security systems are currently separate systems and are liable to monthly fees. Video recording for closed-circuit television (CCTV) is done locally subsequently requiring high storage space. Whenever there is a break-in, the footage records can be stolen consequently losing data. To address high data storage space, monthly premium subscriptions, cost of separate systems and data loss issues of the aforementioned systems, we design and implement a Raspberry-pi based fire and intrusion detection systems in this work. The system sends an SMS in the case of an intrusion or fire detection, and then records and uploads the surveillance videos. The system used a GSM modem for sending SMSs, a video, a PIR sensor to detect motion and a smoke or heat sensor to detect fire. The system is a low cost combined home security and fire detection Raspberry-pi system intended for home and small offices use.
\end{abstract}

\section{KEYWORDS}

Raspberry-Pi, PIR, GSM, Security

\section{INTRODUCTION}

Crime rates are escalating on a daily basis and thieves seem to generate new techniques of robbing people of their property worldwide. On another note with living standards becoming expensive by the day due to inflation, everyone wants to protect their property, even from threats such as fires. Security system products on the market are expensive to setup due to the relative price of the controllers used and they are also subject to monthly subscriptions since they are monitored by security service companies. Fire safety microcontroller systems available are dedicated towards monitoring fire threats only. These products give an alert by only sounding an alarm, which implies a remote home owner will not be alerted by the system when there is a fire outbreak. On the other hand, a fire alarm system is vital to safety. However, it simply triggers an alarm. Fire and burglar alarm system include sophisticated components such as environmental sensors for motion, temperature, panic alarms, beam detection, vibration sensors, glass break detection and many more. Yet, it has a high cost. Though surveillance Digital Video Recorder (DVR) and Network Video Recorder (NVR) consoles now have streaming and motion detection recording features they are expensive to install (online DVR prices average $\$ 100$ on Amazon). Recordings from these systems are normally lost due to damage by thieves or burnt in cases of a fire.

This project seeks to integrate intrusion and fire detection and surveillance camera using a Raspberry-pi microcontroller, which alerts the user by sending a message. This project 
incorporates all these features into one entity and adds extra functionalities such as remote video storage and SMS notifications. There is also data protection assurance as information will be/is stored on the cloud where it is less susceptible to loss. This data protection feature is key for insurance purposes [1].

The rest of this paper is organised as follows. In Section 2, the literature review is presented with the important theory behind the project components covered. Section 3 presents the methodology which covers the block diagrams of the system, the algorithms developed and implemented and the schematic diagrams of the system. Section 4 presents and overview of the code and the test cases used to test the system. Section 5 presents the discussion section and how the system can be improved and used. We conclude the paper in section 6.

\section{LITERATURE REVIEW}

Microcontrollers are standalone devices that are configured to monitor and carry out tasks [2]. They are characterized by features such as memory, processors and input/output pins which allow them to communicate with outside world. There are a number of microcontrollers in the industry of which Arduino is a more common household name due to its utility flexibility. The Arduino is a microcontroller design that is more capable to handle a single task as it has less memory. Raspberry-Pi is however, a microcomputer that has a higher processing power as it has a more powerful processor than the Arduino and has expandable memory[3]. It can literally operate as a computer and have an operating system installed into it which makes it more versatile. The Raspberry-Pi can operate on diverse operating systems such as Raspbian and windows. It uses python to generate code for this microcontroller to operate and interact with the outside world. In this project we used the raspberry-pi due to several reasons. Some to mention are its expandable memory which will allow for video recording and it also has Ethernet port which is in-built [3].

\subsection{Home Safety and Security System}

System integration is basically the process of connecting different subsystems into a single entity with a simplified and centralised management [4]. Literature reviewed shows that security and fire system have been two divergent topics in Botswana, South Africa, Tunisia and in many countries. The lagging behind in the integration of such systems was on the basis of their design requirements. Fire detection system are safety system and tampering threats are not a concern when designing such systems. Design requirements for fire detection systems are proceeds of design regulation, which are overseen by the fire Department in Botswana [5]. In the Botswana factories act it is stipulated that fire detection systems should be accessible to the public, a point which is contrary to security systems design considerations [6]. On the contrary tamper protection is a key feature of consideration for security system design hence having their controllers in locations of public access is a security threat [7]. In Tunisia also separate systems exists such as video surveillance camera and burglar alarm system. However, they require an incident awareness center for a video-centric monitoring and a video intrusion alarm verification [8].

To our knowledge the hindrance to having a single system monitoring safety and security was biased towards businesses and is not considerate of home owners. According to the Botswana Fire Department fire systems inspections based on the fire systems regulation are for business licensing purposes [5]. For households, design is less regulated allowing for a creative and flexible approach towards low cost solutions.

Security and fire detection systems discussed above only give the user an alert but do not portray the status-quo [9]. Available surveillance cameras are not linked to the alarm system and fire 
system. There is therefore, no direct correlation between the detection systems and surveillance system. Monitoring of recorded content is done by human beings physically who can make mistakes due to many reasons [9]. This manual work done by security personnel also implies additional cost on the owners [10]. The Arduino system developed by Kunal Kumar stresses this point, as it effectively alerts a remote user of intrusion and fire through an sms [11]. The system incorporates both smoke and heat parameters to detect fire. However, it does not provide substantial evidence of how detection incidents unfolded.

Surveillance camera solutions available are Network Video Recorder (NVR) and Digital Video Recorder (DVR) [12]. These two solutions store surveillance records locally in a hard-drive. These records are susceptible to loss in occurrence of a fire or damage during break-ins. This loss of information makes the system futile as there will be no evidence for crime investigations. It is also difficult to succeed in insurance claims when there is no concrete proof of the legitimacy of fire causes [13]. These systems continuously record videos, a feature which is not economic in terms of memory preservation.

It is therefore, imperative that work can still be done on remote recording and cohesion of information from fire and security alert systems into a more robust surveillance camera system.

The Raspberry-pi fire detection system by Rosni Abu Kassim is interfaced with and Arduino for connecting the GSM modem which translates into higher power consumption and cost [14]. The overhead in this project is unnecessary as the raspberry-pi is capable of handling these functionalities without an Arduino.

The security system developed by Singoee Sylvestre $\mathrm{S}$ which is also based on the raspberry pi was capable of motion detection and alerting a remote user through email [15]. However the project could not archieve video uploading into the internet. Open-CV was used for video upload in the project. It also has motion tracking functionality which is unnecessary. There is therefore a need to develop a system that is capable of uploading videos online .

The systems discussed above are separate systems for fire and motion detection. Work done by Ashwini Pawar incorporates both motion and fire detection [16]. The system captures images upon detection and emails them to the user. Email is however an internet based which has limited coverage as compared to mobile networks for alerting the user. Photos sent by the system do not communicate sufficient details of a detection scenery unlike a video which is more detailed.There is therefore need to develop an integrated motion, fire and surveillance system which will result in lower maintenance cost since there is only a single central controller [17]. There is also potential energy saving as the power that could have been exhausted by distinct dedicated microcontrollers is relatively less.

\subsection{Review of Hardware and Cloud Storage}

\subsubsection{Smoke and Heat Sensors in Fire Detection Application}

Smoke detectors give early detection for fires that develop slowly. Heat is needed to propagate the smoke towards the detector which is normally mounted on the ceiling [18]. A small amount of heat from smouldering objects is sufficient to lift smoke towards a smoke detector, triggering an alarm before flames are initiated.

Heat detectors operation on the other hand is dependent on temperature change [18]. The amount of heat needed to activate the heat sensor is dependent on the distance of the sensor from the floor. It has been shown that when a flame reaches a height of about one third of that of the ceiling, it can be detected by heat detector. The type of fire determines which detector is more 
suitable. A fire that starts off with smoke will be more accurately detected by a smoke detector and a rapidly flaming fire by a fire detector. A fire detection system developed by Kunal Kumar incorporates features of both sensors by the use of a heat and smoke sensor for more accuracy [11]. If either or both the sensors are triggered the system equates it to a fire. There are however newer sensors which have both properties embedded in one sensor [19].

\subsubsection{Pi-Camera}

Raspberry has a Pi-cameras which can be included into their systems. This camera connects to the microcontroller through a ribbon connector [20]. The ribbon connector attaches to a customized connector in the raspberry-pi.

\subsubsection{Passive Infrared Sensor (PIR)}

Passive infrared motion sensors (PIR) operate by detecting levels of infrared radiation [21]. PIR's are small, inexpensive and low power devices, just the properties that render them attractive for motion sensing. In order to enlarge the coverage of the sensor a plastic lens is used which basically condenses the room into small area. The lens used is termed Fresnel lens and it works as a radiation condenser.

\subsubsection{MQ-135 Smoke Sensor}

The MQ-135 gas sensor can detect smoke. MQ-135 has both digital and analogue output as shown in figure 1. The digital output goes high whenever a threshold of the unwanted gas is reached and gives a low when there is no gas[22].

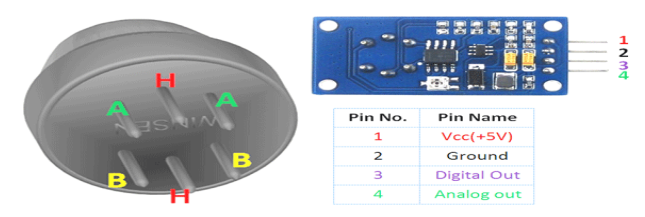

Figure 1: MQ-135 gas sensor connection pins [23].

\subsubsection{DHT-11 Heat Sensor}

DHT-11 is a dual sensor for humidity and temperature. The sensor has 3 pins for power and digital data. The sensor signal is a digital signal which has been calibrated to give accurate readings of temperature and humidity [24].

\subsubsection{Piezo Buzzer}

Piezo buzzer are electrical devices that produce sound [25].The piezo buzzer operate by the inverse of the piezo principle i.e. produce sound when electricity is applied to them.

\subsubsection{Cloud Storage}

There are many online storage platforms available with different free and paid services. G-mail is the most versatile and has better offer in terms of free memory and value for money in paid storage service. Cloud storage services do not support ARM based devices of which raspberry pi is based on [26]. However, there has been developments to solve this problem. G-Rive is one of the solutions for a desktop email app in raspberry pi. It however, involves complex steps to set up 
and there is a charge attached. This fact makes Dropbox more attractive to work with since there is sufficient developments in Adafruit aiding it to be used in raspberry pi as well as it is free. In this work, dropbox was used to store videos online in the cloud.

\subsubsection{GSM Modem}

Global System for Mobile Communication modems (GSM modems) are specialized modems which function by subscription to a mobile network [27]. Since the Raspberry-pi operates just like a computer they can be consolidated into the system for access into the mobile network. Mobile networks are more reliable than internet for alerting a remote home owner. In this work we used SIM cards for Orange and Mascom mobile networks in Botswana.

\section{Methodology}

The raspberry-pi microcontroller was used for this project as there are a number of tasks to be handled which call for a more sophisticated and robust processing power. Figure 2 shows a pictorial overview of the system functions. The system detects fire and motion. It has a camera which is activated in correlation with detection. The raspberry-pi then process the detection signals and output to the phone, speaker and dropbox accordingly.

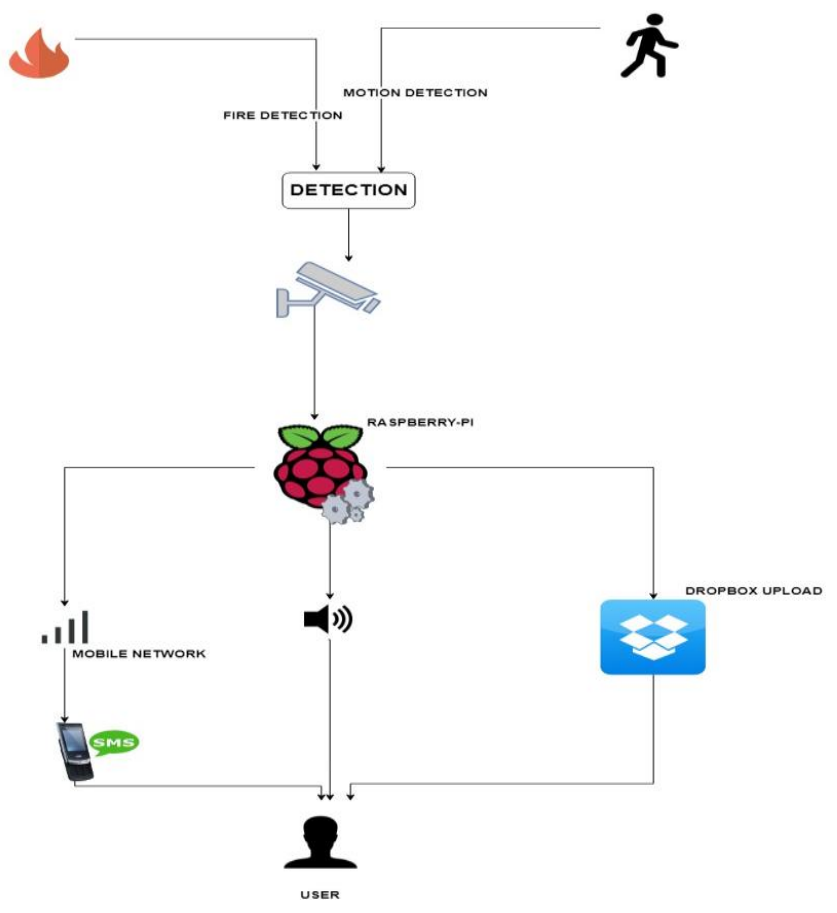

Figure 2: showing a pictorial view of a raspberry-pi based security and safety system. 
International Journal of Computer Science \& Information Technology (IJCSIT) Vol 11, No 3, June 2019

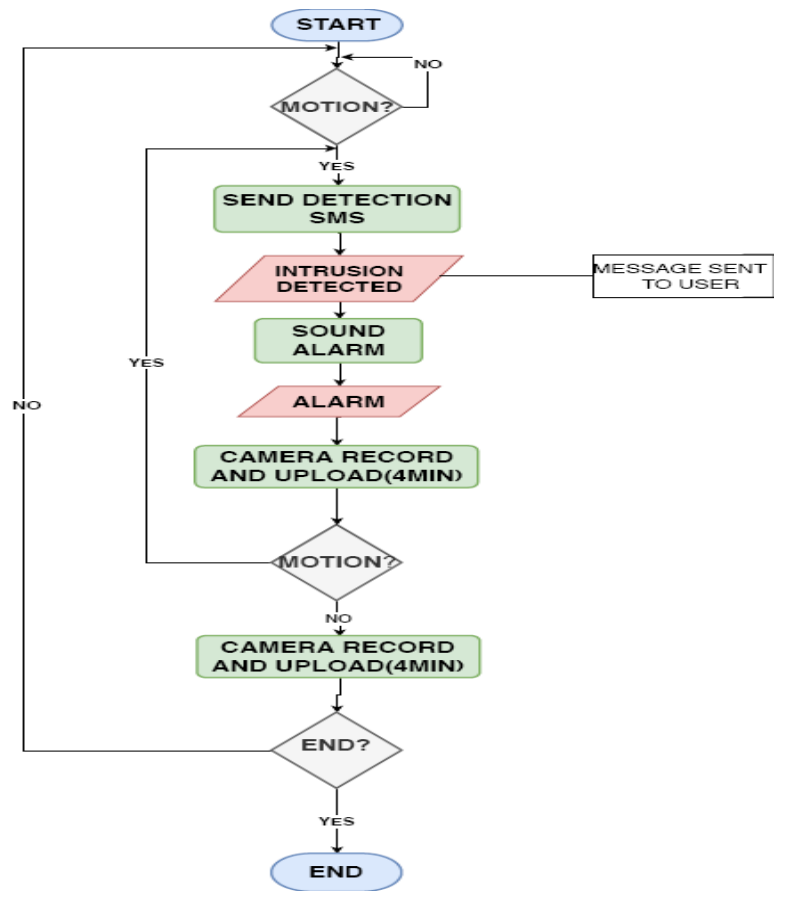

Figure 3: Motion/intrusion detection flowchart

The system checks if there is motion, upon detection an intrusion detection is sent to the user. The video of the scene is recorded and then uploaded in the cloud. If there is no longer motion the system records the last video and goes back to sensing motion else it loops back to the intrusion outputs.

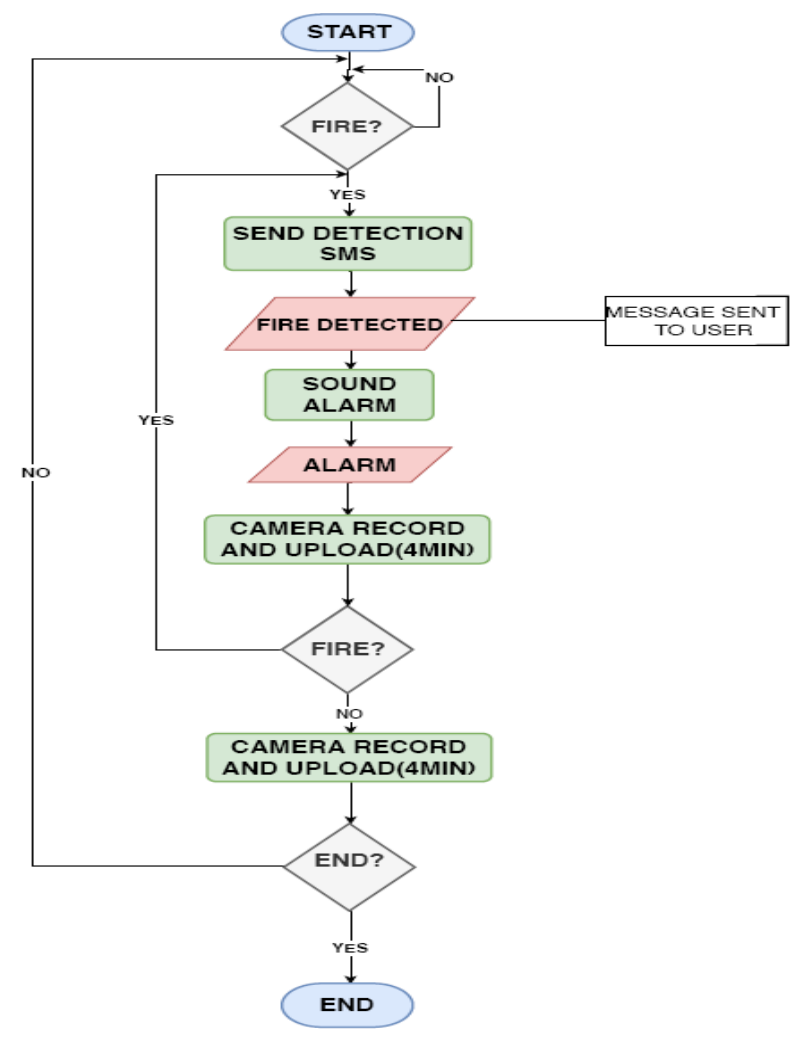

Figure 4: Fire detection system flowchart 
The system checks if there is fire detected, upon detection a fire detection alert is sent to the user. The video of the scene is recorded and then uploaded in the cloud. If there is no longer motion the system records the last video and goes back to sensing fire else it loops back to the fire detection outputs. Figure 5 presents the integrated complete system flowchart. The integrated flowchart is made up of the functionalities of the motion and fire detection described above.

The pseudo code for the motion detection and fire detection systems is given below:

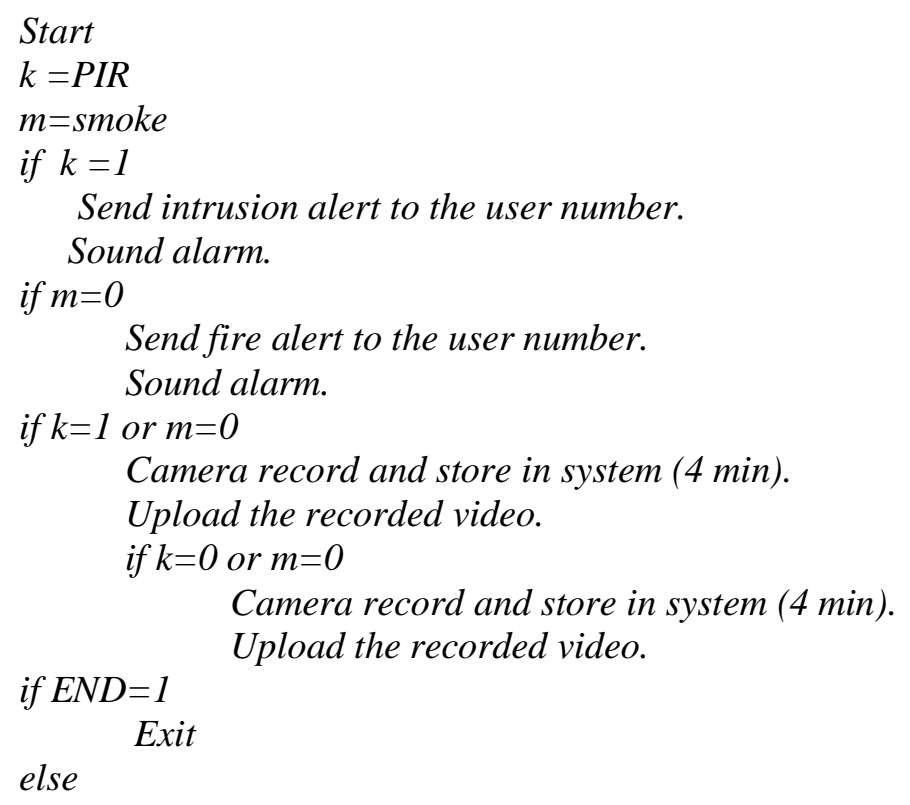

Return to start.

Figure 6 presents the schematic diagram of the complete system. 
International Journal of Computer Science \& Information Technology (IJCSIT) Vol 11, No 3, June 2019

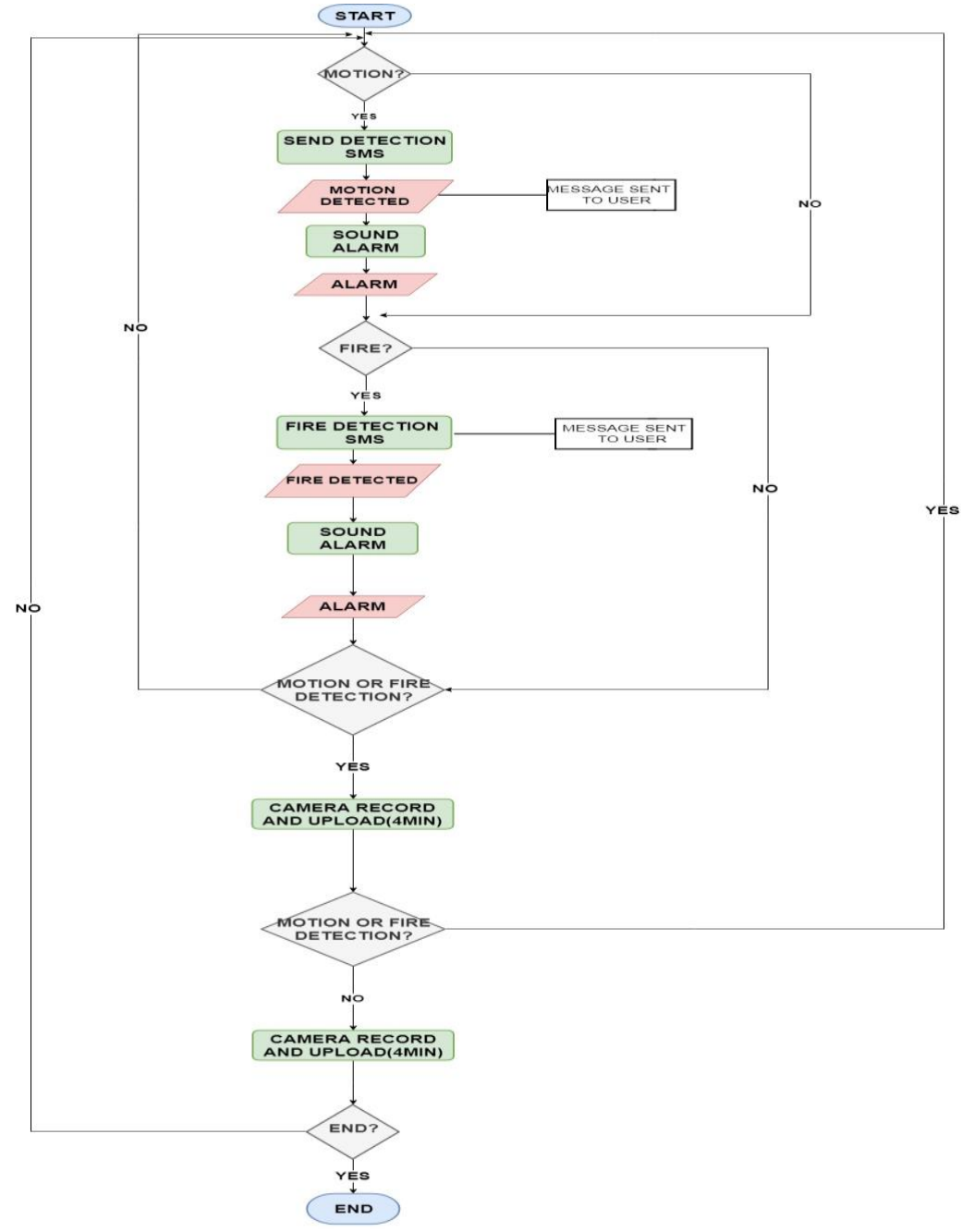

Figure 5: Integrated motion and fire detection system flowchart.

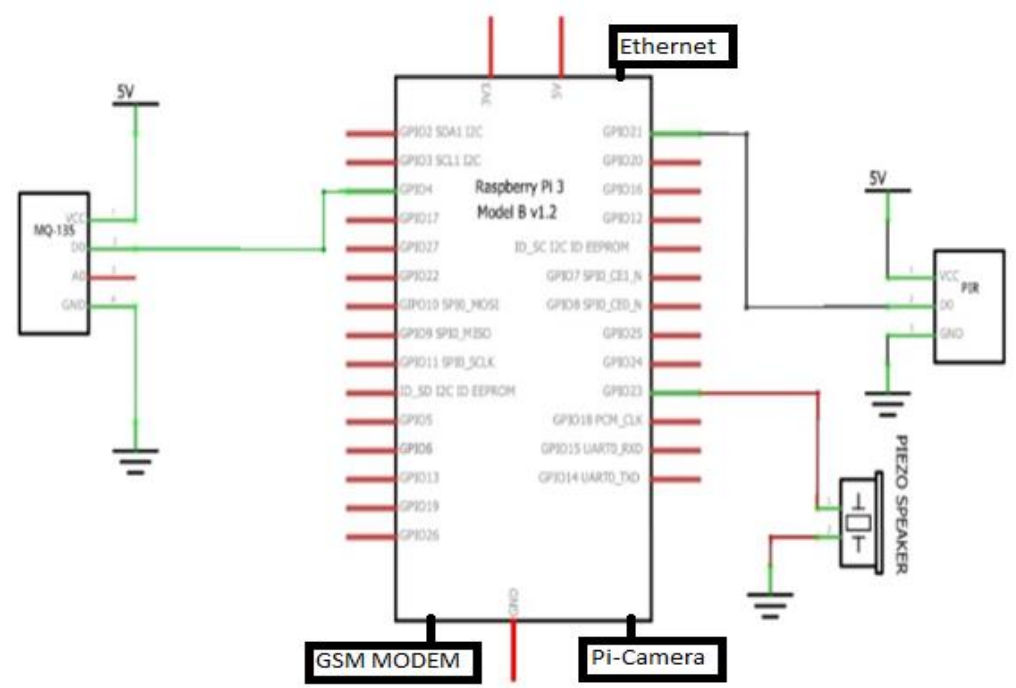

Figure 6: Schematic diagram of the system. 


\section{RESUlts}

In this section we present the overview of the code and the test cases used to test the system.

\subsection{Code Functionalities}

\subsubsection{Motion Detection}

A variable was assigned to the digital input in the GPIO pins from the PIR sensor. This variable was then used as a condition for triggering intrusion alert and the video features of the system. In Code 1 extract below the signal variable for the PIR sensor is ' $\mathrm{i}$ ' and whenever it is high the message is sent.

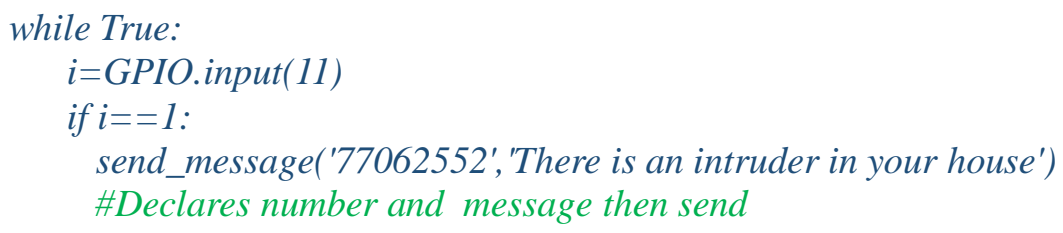

\subsubsection{Fire Detection}

DHT-11 library from Adafruit was imported to aid reading the DHT-11 sensor. The temperature reading is assigned to a variable as a condition for sending the message at a boundary of $41^{\circ}$ (see code extract 2 below). The temperature boundary was chosen based on the average maximum temperature $\left(40^{\circ}\right.$ for Botswana). To effective detect fire, a smoke and heat sensor are proposes to be used in combination.

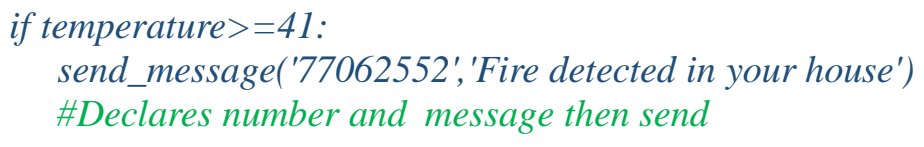

\subsubsection{GSM Modem}

In order to set-up communication between the GSM modem and raspberry-pi, picocom software was installed. A function was defined outside the main part of the code to define the message sending commands. The function was thereafter called within the main code. The message sending code uses AT command. Whenever it is called for both the motion and fire detection there is a recipient number entry and the message field as shown in the code extract below. The GSM simcard in the GSM modem is loaded with airtime to send messages.

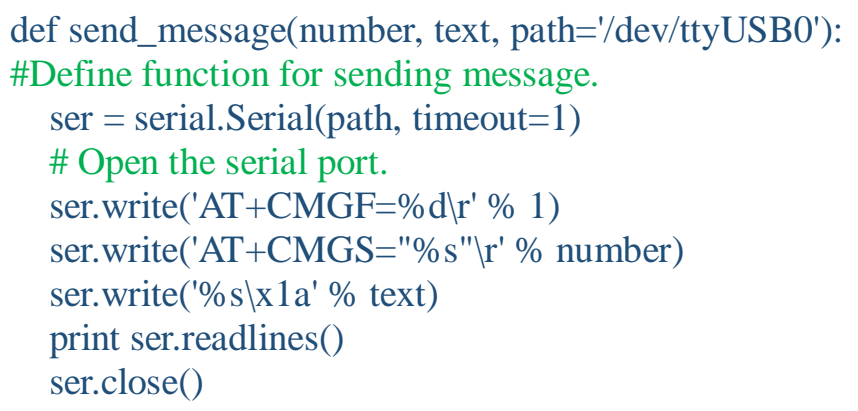




\subsubsection{Buzzer}

The signal or input voltage for the buzzer was connected to a GPIO pin declared as output. In order to generate sound the pin is set high for a few seconds and then off. This pin is assigned a variable 'buzzer' which is then used to set high and low as shown in the code extract below.

GPIO.output(buzzer, True)

time.sleep (.1)

GPIO.output(buzzer, False)

time.sleep (.2)

\subsubsection{Camera}

Pi-camera video recording dimensions are declared in the code. A date-stamp is attached to each video recorded such that it will be easy to identify the date and time of events. Date-stamp is in the format 'year-month-day-hour-minute-second'. The video length is timed to allow for uploading and checking if there is still any signal from the sensors. For this project video recording time is 4 minutes. Having the videos in portions make it easier to manage and preserves cloud storage as whenever there is no activity the recording and uploading seizes. Videos are recorded in an '.h264' format which can be played by a VLC media player in a computer. In the 'camera.start_recording' command, the file path in which the video is to be saved is defined. If there is no detection after recording and upload the system records

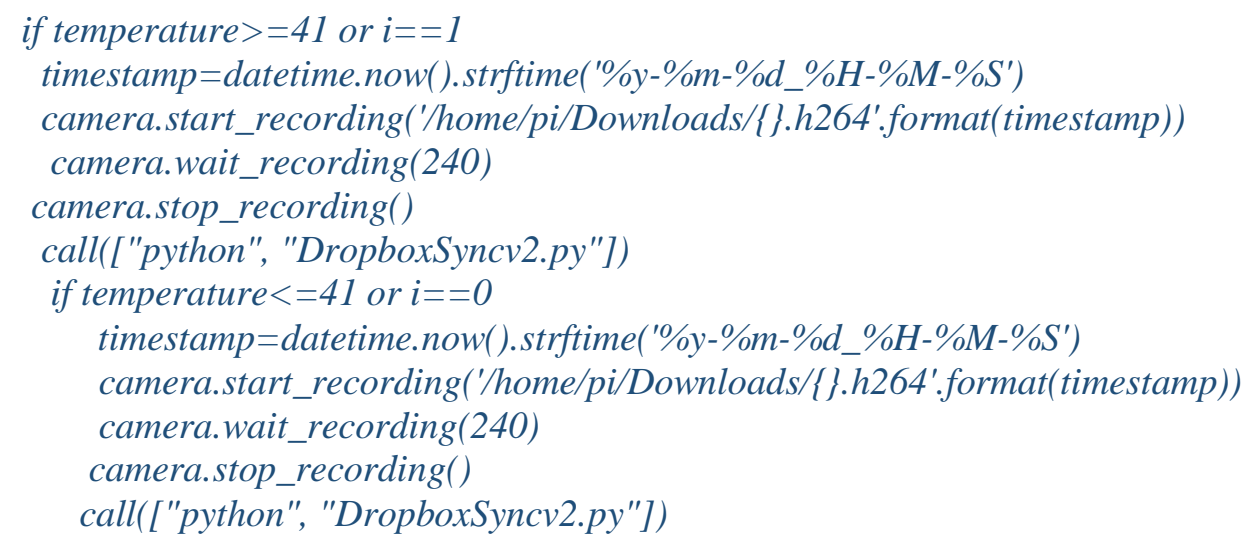

\subsubsection{Dropbox Uploading}

A free 2GB Dropbox account was created for the system. The account can always be upgraded by paying a monthly or yearly premium with memory of up to 1TB. In order for authorization into the account a Dropbox APP was developed. Dropboxsyncv2 script was imported to aid the upload into Dropbox. The script is saved in a local folder to be synched. An access token which is generated through the APP is stored in this local folder as text file (.txt extension). The file path for saving recorded videos mentioned in the camera section above is the same as that of the access token. Whenever this script is run all the files in this folder are uploaded into Dropbox (see code extract below).

$$
\text { call(["python", "DropboxSyncv2.py"]) }
$$

The python code was written in Geany IDLE and in order to run it had to be done manually on a desktop setup. In order to reduce the monitor, mouse and keyboard overhead the script was set to run at startup. This was achieved by the crontab method described above in theory. 


\subsection{Test Cases}

Three test cases were setup to detect the units separating and then the complete system with both functionalities integrated.

\subsubsection{Motion}

Whenever there was motion within the range of the PIR sensor an alerting message was sent. Motion also triggered the buzzer to sound and started the camera to record. The recorded videos was then uploaded to Dropbox (see Figure 7 below). Whenever motion within the range of the PIR sensor seized the system recorded and uploaded one more video and returned to the sensing status. Sensing status means when none of the system outputs are triggered and it is on guard (listening) through the sensors for any triggering signals. Wired LAN connection to the raspberry pi was used.

\subsubsection{Fire}

In order to test for fire, temperature within the range of the DHT-11 was raised to $41^{\circ}$ and above by introducing a hot body within range of the sensor. The system responded by sending an sms (see Figure 8) and then sounding a buzzer and ultimately recording and uploading a surveillance video (See Figure 8). Normalising temperatures within reach of the DHT-11 sensor (temp $<=41$ ) the system recorded and uploaded one more last video (4 min) and returned to the sensing status.

\subsubsection{Combined System with motion and fire}

Whenever there was motion within the range of PIR sensor and temperature was raised above the set $41^{\circ}$ the following outputs were observed respectively;

a) Intrusion detection sms and a buzzer sound.

b) Fire detection sms and a buzzer sound.

c) Camera record and upload for $4 \mathrm{~min}$.

d) Stopping the sensor triggering instances i.e. motion and temp $>=41$ the system recorded and uploaded one last video returned to the sensing status.

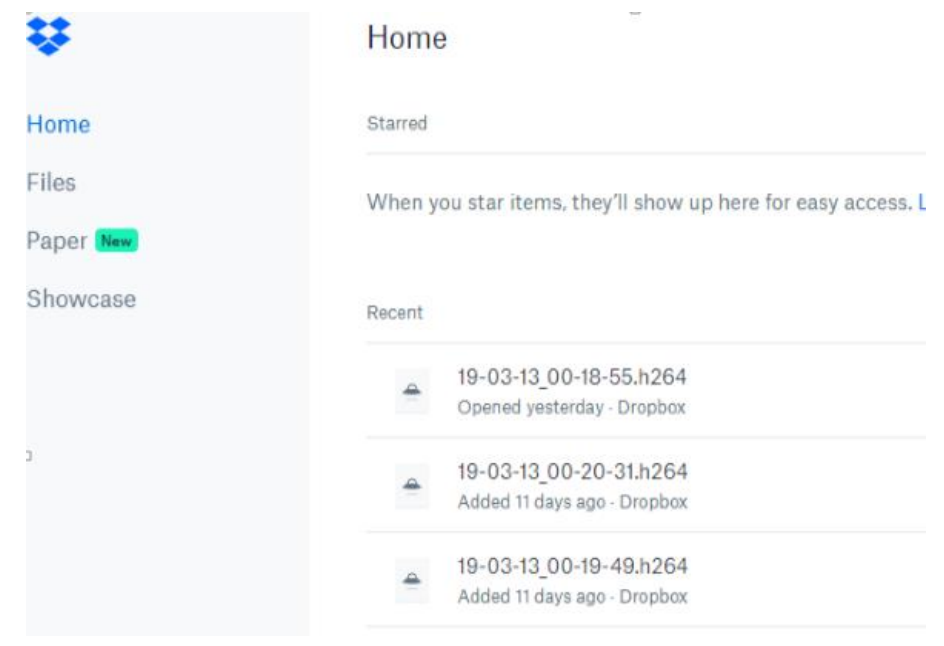

Figure 7: Surveillance videos uploaded in Dropbox named with a date-stamp. 


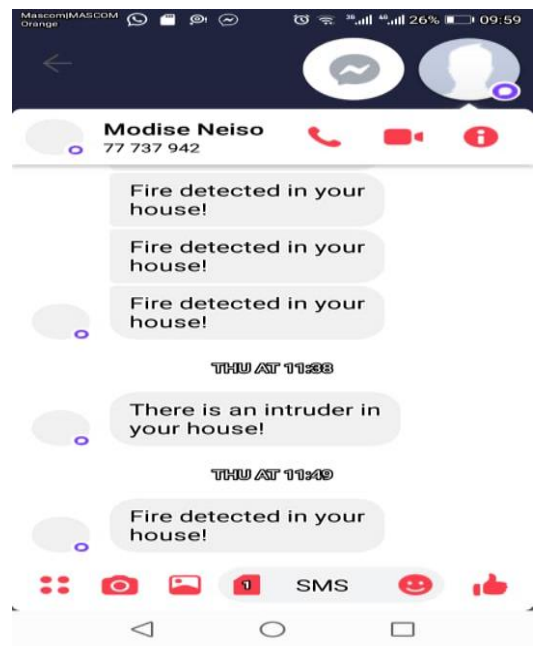

Figure 8: Fire and motion alert SMS sent by the system.

\section{DISCUSSION}

Videos were only recorded when there was detection of with motion or fire. This feature helps preserve both the local and cloud storage space. The uploaded videos have a date-stamp attached to them in the format (year-month-day-hour-minute-second) specified in the python script and it was consequently easy to reconcile detection messages against their respective videos. The videos for all the detections were uploaded since Ethernet connection which is more reliable was used. Each recording session upon detection was limited to 4 minutes which reduces the risks of losing data in terms of connectivity interruptions. This is because videos with a longer time-span takes longer to upload which increases the risk of upload failure consequently increasing the probability of data loss. In the instance when there is no motion or fire detection prior to a video record and upload the system recorded and uploaded the last video and went into a sensing mode.

Fire detection functionality can be made more accurate by coupling both smoke and heat variations to determine fire occurrences. Since the raspberry-pi has a number of GPIO pins the system can be expanded to monitor a number of locations in premises. More motion and smoke sensors can be added to the system depending on the size of the house, rooms etc. Also IP wireless cameras can be incorporated into this expansion for surveillance.

\section{Conclusion}

The Raspberry-pi has shown to be effective in the successfully design and implementation of a fire and intrusion detection system for the home or small businesses with video recording and uploading functionalities. The system is able to send SMS alerts, video recordings and upload upon detection. Although the system meets the project objectives, there is still fine tuning and improvement of the system ongoing. This includes development a battery management system for long live and operation when there are power cuts. The coupling of smoke and heat detection sensors can make a fire detection more accurate and to trigger when there is a fire. In order to prevent exhaustion of memory the system can be set-up to automatically delete old videos on a regular basis e.g. monthly.

Video recordings are vital for insurance claims and evidence of events. The recording when necessary feather allows saving in storage space. Also the cloud uploading allows the files to be 
saved to be more secure, making it less possible to loose files due to theft and damage. In a nutshell, the system proves to be a low cost system to combine security and fire detection features.

\section{REFERENCES}

[1] "Undetermined Fire [Online]. Available: http://clmmag.theclm.org/home/article/Undetermined Fire Losses. [Accessed: 05-May-2019].

[2] G. Gridling and B. Weiss, "Introduction to Microcontrollers," 2007.

[3] R. Zwelsoot, VOLUME 2 PROJECTS BOOK FROM THE MAKERS OF THE OFFICIAL RASPBERRY PI MAGAZINE of coding \&amp; creating 200 PAGES. Cambridge: Raspberry Pi (Trading) Ltd.

[4] Karri Lehtonen, "What is system integration?" [Online]. Available: https://www.youredi.com/blog/what-is-system-integration. [Accessed: 12-Apr-2018].

[5] "Republic of Botswana - Government portal," 2011. [Online]. Available: http://www.gov.bw/en/Ministries--Authorities/Local-Authorities/Jwaneng-Town-Council/Tools-andServices/Services/FIRE-DEPARTMENT/. [Accessed: 24-Aug-2018].

[6] T. National Assembly of Botswana and the C. Botswana Government Printer Exploitation right sin the are held by Juta Law with the approval of the Government of Botswana, "LAWS OF BOTSWANA."

[7] "Intrusion Detection System," 2011. [Online]. Available: https://shareng.sandia.gov/itc/assets/08_intrusion-detection.pdf. [Accessed: 15-Sep-2018].

[8] "inel Security.".

[9] V. Shukla, G. K. Singh, and D. P. Shah, "Automatic Alert of Security Threat through Video Surveillance System,” no. November, 2015.

[10] BSIA, “A Guide to Integrated Security Management Systems,” 2007.

[11] K. Kumar, N. Sen, S. Azid, and U. Mehta, "A Fuzzy Decision in Smart Fire and Home Security System,” Procedia Comput. Sci., vol. 105, pp. 93-98, Jan. 2017.

[12] "DVR vs. NVR - What's the Difference? | Swann Security." [Online]. Available: https://www.swann.com/blog/dvr-vs-nvr-whats-the-difference/. [Accessed: 18-Oct-2018].

[13] "Claims explained." [Online]. Available: http://understandinsurance.com.au/claims-explained. [Accessed: 01-Oct-2018].

[14] M. Saifudaullah and B. Bahrudin, Development of Fire Alarm System using Raspberry Pi and Arduino Uno. .

[15] S. S. Sheshai, "University of Nairobi Department of Electrical and Information Engineering Raspberry Pi Based Security System Prj Index 156,” 2016.

[16] A. Pawar and V. M. Umale, "Internet of Things Based Home Security Using Raspberry Pi," in 2018 Fourth International Conference on Computing Communication Control and Automation (ICCUBEA), 2018, pp. 1-6. 
International Journal of Computer Science \& Information Technology (IJCSIT) Vol 11, No 3, June 2019

[17] C. Lewis, "Fire and security products 2016," 2016. [Online]. Available: https://www.chrislewis.co.uk/fireandsecurity/wp-content/uploads/sites/2/2016/04/The-real-benefitsof-fire-and-security-integration.pdf. [Accessed: 03-Apr-2019].

[18] P. G. Smith, "Fire-detection and alarm systems," Wiring Install. Supplies, vol. 1977, no. 3, p. 9, 2010.

[19] "Smoke Alarms - UK Fire Service Resources." [Online]. Available: https://www.fireservice.co.uk/safety/smoke-alarms/. [Accessed: 05-May-2019].

[20] S. Monk, Raspberry Pi Cookbook. 2016.

[21] A. Industries, "PIR motion sensor," US Pat. 7,579,595, 2009.

[22] Waveshare, "MQ-135 Gas Sensor User Manual." [Online]. Available: https://www.robotshop.com/media/files/pdf/MQ-135-Gas-Sensor-UserManual.pdf. [Accessed: 05May-2019].

[23] Components101, "MQ-135 Gas Sensor Pinout, Features, Alternatives, Datasheet \&amp; Uses Guide," 2018. [Online]. Available: https:/components101.com/sensors/mq135-gas-sensor-for-airquality. [Accessed: 05-May-2019].

[24] "DHT11 Sensor Pinout, Features, Equivalents \&amp; Datasheet." [Online]. Available: https://components101.com/dht11-temperature-sensor. [Accessed: 05-May-2019].

[25] "Insight - Working Principle of Piezo Buzzer | How Piezo Buzzer works." [Online]. Available: https://www.engineersgarage.com/insight/how-piezo-buzzer-works. [Accessed: 05-May-2019].

[26] “OwnCloud on Raspberry Pi." [Online]. Available: https://eltechs.com/owncloud-on-raspberry-pi/. [Accessed: 05-May-2019].

[27] "What is a GSM Modem? | NowSMS." [Online]. Available: https://www.nowsms.com/faq/what-isa-gsm-modem. [Accessed: 05-May-2019].

\section{AUTHORS}

Dr. Sajid M. Sheikh is an academic, researcher and consultant. He is currently a Senior Lecturer in the Department of Electrical Engineering, Faculty of Engineering and Technology, University of Botswana. He is also the MSc Coordinator in the Department of Electrical Engineering, Faculty of Engineering and Technology, University of Botswana, Cisco Instructor at the UB-FET Cisco Academy at University of Botswana and IEEE Secretariat for the Botswana IEEE Subsection. Dr. S. M. Sheikh holds qualifications of $\mathrm{PhD}$ in Electrical Engineering from University of Stellenbosch (South Africa), MSc in Electronic Systems Engineering from the University of Botswana, BEng in Electrical and Electronic from the

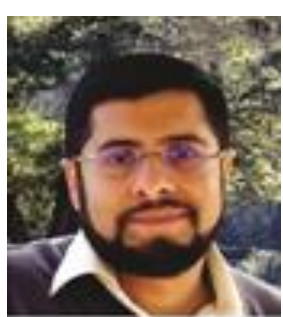
University of Botswana, CCNA 1, 2, 3 and 4 Instructor qualification Courses from University of Botswana Cisco Academy, IT Essentials Instructor qualification Course from Sci-Bono ICT Academy in New Town, Johannesburg and IT Essentials Instructor Training Qualification from the Networking Academy Instructor Trainer Cisco Systems (South Africa), South Africa. He also holds professional memberships of Institute of Electrical and Electronic Engineers (IEEE) as a Senior Member and Botswana Institute of Engineers (BIE) as a member. He is a registered Professional Engineer (PrEng) with Engineering Registration Board (ERB) (Botswana) in the discipline of Electrical and Electronic Engineering. He is also an accredited Assessor with Botswana Qualifications Authority. He is an author of many international journal papers, international peer reviewed conference papers and book chapters. He has been / is the reviewer of many international conferences such as IEEE AFRICON 2017; International Conference on Information Society and Smart Cities (ISC 2018); International Conference on Mobile Systems and Pervasive Computing (MobiSPC-2017, 2018); Southern Africa Telecommunication Networks and Applications Conference (SATNAC) for 2017, 2018 and 2019 and so on. 
International Journal of Computer Science \& Information Technology (IJCSIT) Vol 11, No 3, June 2019

Mr. Modise K Neiso is a final year student in the BENG Electrical and Electronic Engineering at the University of Botswana. His strong areas are in digital communications, computer networking and digital systems design engineering applications. His research interests are in Internet of Things, precisely Smart Homes is my interest.

Dr, Fatma Ellouze recieved her $\mathrm{PhD}$ in Computer Science from the Faculty of Economics and Management of the University of Sfax, Tunisia, in September 2018. She is a member of the Multimedia, Information systems and Advanced Computing Laboratory, since 2013. Her current research interests include Business process management, Process modeling, Context Modeling, Ontologies and Information systems.
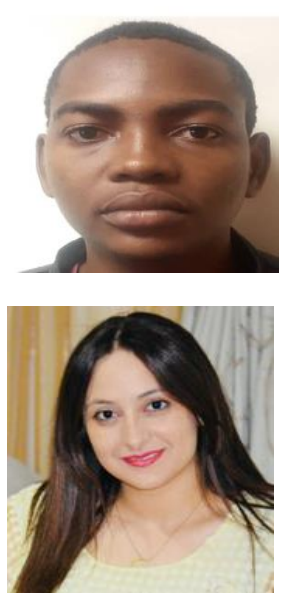